\title{
Quantitative Spatiotemporal Chemical Profiling of Individual Lipid Droplets by Hyperspectral CARS Microscopy in Living Human Adipose-Derived Stem Cells
}

\author{
Claudia Di Napoli, ${ }^{\dagger}$ Iestyn Pope ${ }^{\dagger}$ Francesco Masia, $^{\ddagger}$ Wolfgang Langbein, ${ }^{\dagger}$ Pete Watson, \\ and Paola Borri* ${ }^{\dagger}$ \\ 'School of Biosciences, Cardiff University, Cardiff CF10 3AX, United Kingdom \\ ${ }^{\ddagger}$ School of Physics and Astronomy, Cardiff University, Cardiff CF10 3AX, United Kingdom
}

Supporting Information

\begin{abstract}
There is increasing evidence showing that cytosolic lipid droplets, present in all eukaryotic cells, play a key role in many cellular functions. Yet their composition at the individual droplet level and how it evolves over time in living cells is essentially unknown due to the lack of suitable quantitative nondestructive measurement techniques. In this work, we demonstrate the ability of label-free hyperspectral coherent anti-Stokes Raman scattering (CARS) microscopy, together with a quantitative image analysis algorithm developed by us, to quantify the lipid type and content in $\mathrm{vol} / \mathrm{vol}$ concentration units of individual lipid droplets in living human adipose-derived stem cells during differentiation over 9 days in media supplemented with different fatty acids.
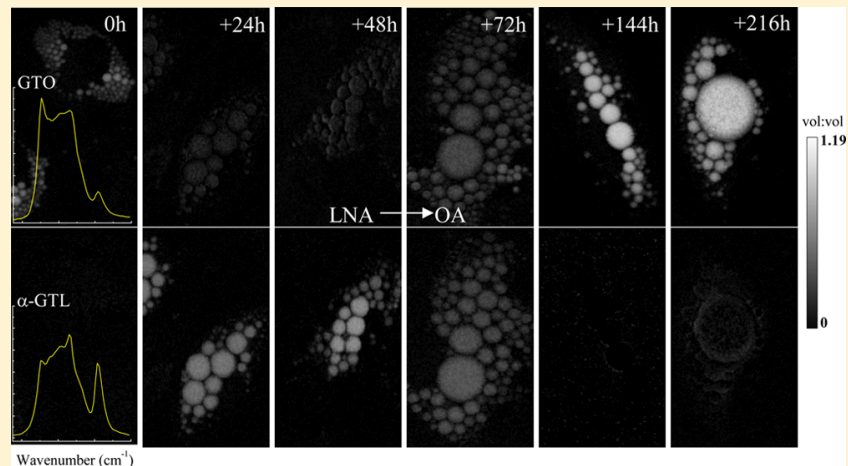
Specifically, we investigated the addition of the polyunsaturated linoleic and alpha-linolenic fatty acids into the normal differentiation medium (mostly containing monounsaturated fatty acids). We observe a heterogeneous uptake which is dropletsize dependent, time dependent, and lipid dependent. Cells grown in linoleic-acid-supplemented medium show the largest distribution of lipid content across different droplets at all times during differentiation. When analyzing the average lipid content, we find that adding linoleic or alpha-linolenic fatty acids at day 0 results in uptake of the new lipid components with an exponential time constant of $22 \pm 2 \mathrm{~h}$. Conversely, switching lipids at day 3 results in an exponential time constant of $60 \pm 5$ h. These are unprecedented findings, exemplifying that the quantitative imaging method demonstrated here could open a radically new way of studying and understanding cytosolic lipid droplets in living cells.
\end{abstract}

$\mathrm{T}^{\mathrm{s}}$ he ability to quantify in absolute concentration units the spatiotemporal profile of chemical components in living cells, without staining or sample preparation artifacts, is still a very challenging task. On the one hand, there are analytical methods such as chromatography, able to quantitatively measure the concentration of, for example, specific lipid types from a cell extract. However, they are invasive, expensive, and timeconsuming and provide only ensemble averages at fixed time points. On the other hand, optical microscopy methods can provide high spatial and temporal resolution in living specimens, but they tend to be qualitative and often require labeling for chemical specificity.

Spontaneous Raman microspectroscopy is gaining increasing attention as a chemically specific optical technique that is able to distinguish the chemical composition of endogenous components in cells through their vibrational spectra, without the need for labeling, and hence free from staining artifacts. Combined with appropriate data analysis algorithms, Raman microspectroscopy is also quantitative. For example, Schie et al. ${ }^{1}$ recently performed a comparative Raman spectroscopy and gas chromatography study of cytosolic lipid droplets (LDs) in HepG2 cells. By utilizing a least-squares fitting procedure of measured Raman spectra with a basis of Raman spectra from pure fatty acids, they were able to quantitatively determine the percentile amount of fatty acids of interest in cytosolic lipid droplets individually resolved with Raman microspectroscopy, which were in good agreement with the ensemble average values from gas chromatography. However, spontaneous Raman scattering suffers from low signal levels that can be easily obfuscated by autofluorescence in biological specimens and usually requires long acquisition times often not compatible with observing living cells. In fact, only fixed cells were studied in ref 1.

Over the past decade, coherent Raman scattering (CRS) microspectroscopy has emerged as a powerful multiphoton microscopy technique which overcomes the signal limitations

Received: November 25, 2015

Accepted: March 2, 2016

Published: March 3, 2016 
of spontaneous Raman scattering through the constructive interference of Raman scattered light from coherently driven molecular vibrations. It also benefits from an intrinsic threedimensional resolution owing to its nonlinearity. Chemically specific label-free imaging of living cells with high spatial and temporal resolution has been demonstrated by CRS microscopy on many biological samples, with particular emphasis on imaging lipids owing to the large number of identical $\mathrm{CH}$ bonds in their acyl chains. ${ }^{2}$ Initial demonstrations of CRS microscopy focused on showing high-speed imaging up to video rate ref $^{3,4}$ but with limited chemical information provided solely from detecting at a single $\mathrm{CH}$-stretch vibrational resonance and unable to give a quantitative chemical map of the lipid concentration. Subsequent implementations of multiplex or hyperspectral CRS imaging, where a vibrational spectrum is measured for each spatial point, enabled a much higher chemical specificity. ${ }^{2,5}$ However, only few works have combined the acquisition of broadband CRS data with a quantitative decomposition analysis, resulting in spectra and spatially resolved chemical maps of separated chemical components. ${ }^{6}$ Many papers only show CRS images where different vibrational resonances are simply being color-coded. ${ }^{5}$ Furthermore, most broadband CRS imaging works so far have been on fixed cells or frozen/dried tissues. $^{7,8}$

We recently developed a powerful data analysis algorithm, which we named "Factorization into Susceptibilities and Concentrations of Chemical Components" (FSC ${ }^{3}$ ), and applied it to label-free hyperspectral coherent anti-Stokes Raman scattering (CARS) images taken with our latest-generation home-built multiphoton microscope. With this system, we were able to provide quantitative chemical maps of independently varying chemical components (dominated by water, proteins, saturated, and unsaturated lipids) in cells in their vol/vol concentration units. ${ }^{9-13}$ As a proof of principle, the method was demonstrated on fixed cells, not using the advantage of CRS over spontaneous Raman for live cell imaging applications.

In this work, we demonstrate hyperspectral CARS acquisition and FSC $^{3}$ quantitative analysis of the lipid composition and concentration in live human adipose-derived stem cells (ADSCs) during differentiation over 9 days after induction of adipogenesis in media supplemented with different fatty acids. Human ADSCs have been the subject of a few CRS imaging studies recently; however, these focused either on how the number and size of LDs change during differentiation, eventually leading to one large unilocular LD at mature stage, ${ }^{14}$ or on how early markers of differentiation into adipocytes can be distinguished. ${ }^{15}$ There is no quantitative imaging of the lipid composition and concentration of spatially resolved cytosolic LDs in live ADSCs followed over time reported to date, despite the biological importance of such study. As extensively discussed in the literature, adipose tissue is the human body's largest energy reservoir, ${ }^{16}$ and adipocytes represent the majority of cells in this tissue, where triglycerides are stored in cytosolic lipid droplets. The triglyceride composition of the adipose tissue and its relationship with dietary fatty acids has become a key topic of research especially after obesity, and associated diseases have become a modern epidemic. ${ }^{17}$ Notably, obesity and type II diabetes have been directly related to malfunction of $\mathrm{LD}$ synthesis, lipid storage, and degradation. ${ }^{18}$ However, due to the lack of suitable techniques with sufficient spatiotemporal resolution, a major and seemingly trivial aspect of adipocytes remains difficult to assess: the specific fatty acid composition of individual cellular lipid droplets and how this evolves over time, for example during adipogenesis. In this work, we show that hyperspectral CARS acquisition and $\mathrm{FSC}^{3}$ quantitative analysis overcomes this limitation, and this analysis has been applied to measure the concentration and time scales of storage and exchange of specific fatty acids types in live ADSCs.

\section{MATERIALS AND METHODS}

Pure Lipids. Bulk pure (>99\%) oleic acid, a monounsaturated 18-carbon chain with a cis double bond at the ninth position from the first carbon atom (cis-9-octadecenoic acid; OA), linoleic acid, a biunsaturated 18-carbon chain with two cis double bonds at the ninth and 12th position (cis,cis-9,12octadecadienoic acid; LA), $\alpha$-linolenic acid, a triunsaturated 18-carbon chain with cis double bonds at 9, 12, and 15th position (cis,cis,cis-9,12,15-octadecatrienoic acid; LNA), and glyceryl trioleate (1,2,3-Tri(cis-9-octadecenoyl)glycerol; GTO) were purchased from Sigma-Aldrich. Pure (>99\%) $\alpha$-glyceryl trilinolenate (1,2,3-Tri(cis,cis,cis-9,12,15- octadecatrienoyl)glycerol; $\alpha$-GTL) was purchased from Nu-Chek Prep. CARS measurements on bulk pure lipids were performed at $37{ }^{\circ} \mathrm{C}$. Samples were prepared by pipetting $15 \mu \mathrm{L}$ of bulk lipid into a well created by the $9 \mathrm{~mm}$ diameter opening of a $0.12 \mathrm{~mm}$ thick adhesive imaging spacer (Grace BioLabs) attached to a microscope slide, and subsequently capped with a \#1 coverslip.

Cell Culture and Treatment. Human ADSCs purchased from Invitrogen (Carlsbad, CA, U.S.A.) were grown in low glucose Dulbecco's modified eagle medium and GlutaMAX supplemented with $10 \%$ mesenchymal stem cells qualified fetal bovine serum (FBS), $75 \mu \mathrm{g} / \mathrm{mL}$ gentamicin, and $37.5 \mu \mathrm{g} / \mathrm{mL}$ amphotericin. In order to induce differentiation into adipocytes, they were cultured in StemPro adipogenic differentiation media as indicated by the manufacturer's protocol. For experimental purposes, cell cultures were grown directly on glass coverslips (thickness \#1.5, $25 \mathrm{~mm}$ diameter, PA, VWR International, U.S.A.). After 3 days, media were supplemented with fatty acid-bovine serum albumin (BSA) complex $(9.4 \mu \mathrm{g} / \mathrm{mL}$ fatty acid concentration), as the starting point (day 0 ) of subsequent time-course experiments. Fatty acid-BSA complexes were prepared in-house following published protocols. ${ }^{19}$

For live cell imaging, the cell-seeded coverslips were mounted in custom-made stainless steel holders forming a Petri-dish like chamber with a glass lid (in which they remained for the duration of the experiment) and were observed directly from day 0 . Four different samples were prepared consisting of human ADSCs in supplemented media with (i) OA, (ii) LA, (iii) LNA, and (iv) a 1:1 volume/volume mixture of oleic acid and $\alpha$-linolenic acid (all as fatty acid-BSA complexes). A fifth sample was left in the normal differentiation medium and used as a control. After $48 \mathrm{~h}$, the OA supplemented medium was substituted with LNA-supplemented medium and vice versa, and on all other samples, media were exchanged with fresh identical media. After $144 \mathrm{~h}$ (day 6), on all samples media were again exchanged with fresh identical media. Live cell imaging measurements were performed daily up to day 9 . While undergoing imaging, cells were kept at $37{ }^{\circ} \mathrm{C}$ in a $5 \% \mathrm{CO}_{2}$ air blend atmosphere. Between measurements, samples were kept in a tissue culture incubator, again with a 5\% CO2 air blend atmosphere at $37{ }^{\circ} \mathrm{C}$. Alignment marks on the exterior of the imaging chambers ensured their precise repositioning on the microscope stage enabling specific cells to be tracked throughout the course of the experiment.

CARS Microscopy Setup. We performed hyperspectral CARS imaging using the home-built setup described in detail in 
our previous works. ${ }^{10,20}$ Briefly, a single Ti:sapphire 5 fs broadband (660-970 nm) laser system provided pump and Stokes components for the CARS excitation. We spectrally separated the broadband laser output with an appropriate sequence of dichroic beam splitters, resulting in a pump (Stokes) with center wavelength at $682 \mathrm{~nm}(806 \mathrm{~nm})$ and a bandwidth of $65 \mathrm{~nm}(200 \mathrm{~nm})$. A spectral resolution of $10 / \mathrm{cm}$ is achieved through spectral focusing. ${ }^{21}$ CARS spectroscopy across the $1200-3800 / \mathrm{cm}$ vibrational range was performed without adjustments to the laser, by simply changing the relative delay time between pump and Stokes pulses. The excitation pulses were coupled into a commercial inverted microscope stand (Nikon $\mathrm{Ti}-\mathrm{U}$ ) via a home-built beam-scanning head. The microscope was using a $20 \times 0.75 \mathrm{NA}$ dry objective (Nikon CFI Plan Apochromat lambda series), and the CARS signal was collected in forward direction with a $0.72 \mathrm{NA}$ dry condenser, frequency selected by appropriate band-pass filters and detected by a photomultiplier tube (Hamamatsu H7422-40). The resulting CARS spatial resolution (fwhm of the intensity point-spread function) was measured to be 0.6 and $1.1 \mu \mathrm{m}$ in the lateral ( $x y$ ) and axial $(z)$ direction, respectively. ${ }^{20}$ A motorized sample stage enabled $x y$ sample movement and a motorized objective focusing enabled $z$ movement (Prior ProScan III). The microscope stand was also equipped with differential interference contrast (DIC) optics, and cells of interest were first identified with DIC. CARS hyperspectral $x y$ images were acquired in the fingerprint region $(1200-2000 / \mathrm{cm})$ and in the $\mathrm{CH}$ region $(2600-3300 / \mathrm{cm})$ with $5 / \mathrm{cm}$ spectral steps. $x y$ images were acquired using beam scanning with a pixel size of $0.3 \times 0.3 \mu \mathrm{m}^{2}$ and a pixel dwell time of $10 \mu \mathrm{s}$, resulting in $0.9 \mathrm{~s}$ acquisition time for a $90 \times 90 \mu \mathrm{m}^{2}$ single-frequency CARS image. Correspondingly, a typical hyperspectral image stack with 100 spectral points required a total acquisition time of $90 \mathrm{~s}(1.5 \mathrm{~min})$. To avoid prolonged laser exposure, images were acquired at a fixed $z$ determined as the axial position for which most medium-sized (3-5 $\mu \mathrm{m}$ diameter) LDs are equatorially cut such that the lipid content of their inner core is optimally imaged. Nonresonant CARS spectra from glass were also recorded and used for normalization (i.e., measured CARS intensities in each sample were divided by the corresponding nonresonant CARS intensity in glass under the same excitation and detection conditions) in order to correct for the varying temporal overlap of pump and Stokes and to derive a CARS intensity ratio independent of excitation/detection parameters. For the correct quantification of the CARS intensity ratio, backgrounds were measured under the same excitation and detection conditions with pump and Stokes pulses out of time overlap, and they were then subtracted from the measured CARS intensities prior to calculating the ratio.

Data Analysis. Hyperspectral CARS images were noisefiltered with a singular value decomposition (SVD) algorithm on the square root of the CARS intensity to retain only components above noise, as described in our previous work. ${ }^{9}$ CARS intensity ratios were calculated by dividing the backgroundcorrected CARS intensity by the corresponding nonresonant CARS intensity measured in glass under the same excitation and detection conditions. The phase-corrected Kramers-Kronig method $^{9}$ (PCKK) was used to retrieve from the CARS intensity ratio the complex CARS third-order susceptibility (normalized to the nonresonant value in glass), which is linear in the concentration of chemical components. We then used non-negative matrix factorization applied to the imaginary part and the average real part of the susceptibility with an additional concentration constraint (FSC 3 method) to obtain susceptibility spectra of independently varying chemical components and their volume concentrations. Notably, the $\mathrm{FSC}^{3}$ is unsupervised; that is, it does not require prior knowledge of the spectra of chemical components (instead needed as inputs for quantitative multivariate curve resolution analysis reported by others ${ }^{6}$ ) and uses random initial guesses.

\section{RESULTS AND DISCUSSION}

Hyperspectral CARS images and representative spectra as measured and after phase retrieval using the PCKK method ${ }^{9}$ are shown in Figure 1 for human ADSCs cultured in normal

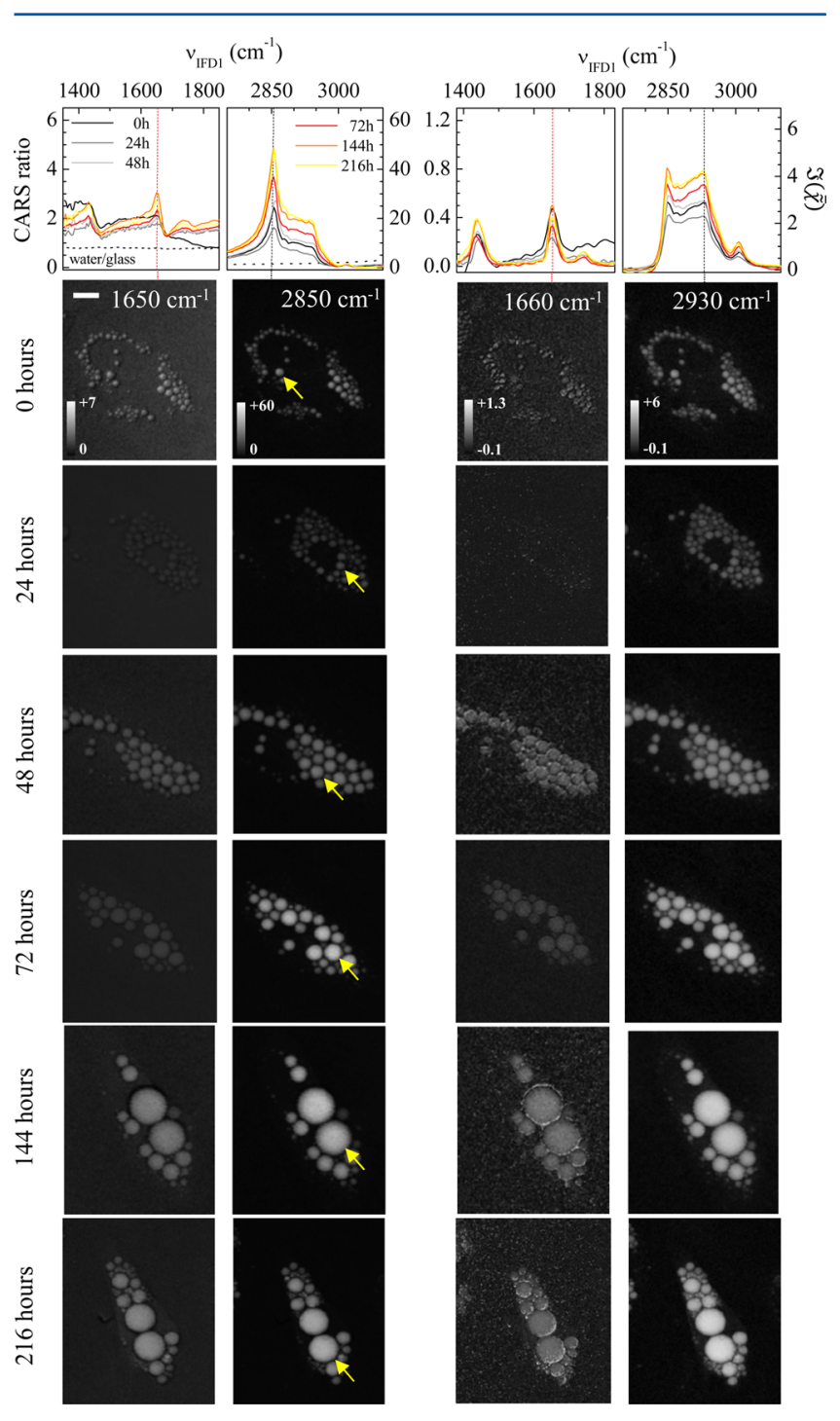

Figure 1. Upper panels: CARS intensity ratio relative to the nonresonant intensity in glass (left) and corresponding spectrum of the retrieved susceptibility (right) at $24,48,72,144$, and $216 \mathrm{~h}$ after day 0 for human ADSCs cultured in control media. Spectra are obtained by averaging over two LDs from cells in two different fields of view. For the displayed field of view, the corresponding $\mathrm{LD}$ is indicated by the yellow arrow. The spectrum of the water-to-glass ratio is also shown. Lower panels: Images of the CARS intensity ratio (left) and corresponding susceptibilities (right) at different time points and wavenumbers, as indicated by the vertical dotted lines in the spectra. Linear gray scales as indicated. The pump power on the sample was $30 \mathrm{~mW}(18 \mathrm{~mW})$, and the Stokes power was $15 \mathrm{~mW}(9 \mathrm{~mW})$ for the fingerprint region (CH-stretch region); $10 \mu$ s pixel dwell time, $0.3 \mu \mathrm{m}$ pixel size, $10 \mu \mathrm{m}$ scale bar. 

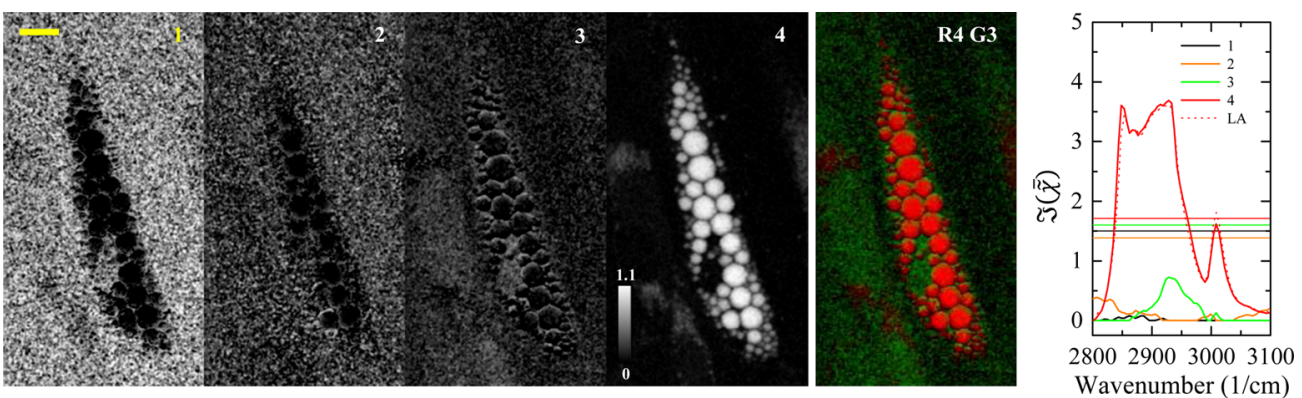

Figure 2. FSC ${ }^{3}$ analysis on human ADSC grown in LA-supplemented media for $72 \mathrm{~h}$ after day 0. Left: spatial distributions of the volume concentration on a gray scale from 0 (black) to 1.1 (white) for the four components considered in the analysis. Scale bar: $10 \mu \mathrm{m}$. Center: red-green overlay showing the spatial distribution of the concentration for component 4 (red) and 3 (green), as indicated. Right: spectra of $\Im(\bar{\chi})$ and its real part (horizontal lines) for the corresponding components. The red dotted line is the $\mathrm{FSC}^{3}$ spectrum measured on pure LA for comparison.

differentiation medium at $24,48,72,144$, and $216 \mathrm{~h}$ after day 0 . CARS intensities are given as ratios relative to the nonresonant response in glass and are therefore independent of excitation/detection parameters (see Materials and Methods section). PCKK allows us to retrieve the imaginary part of the normalized CARS susceptibility $\mathfrak{I}(\bar{\chi})$ which shows a Ramanlike spectrum and is linear in the concentration of chemical components. Representative spectra of LDs (indicated by the arrows) are shown, alongside images at characteristic wavenumbers indicated by the vertical dotted lines in the spectra. CARS spectra show dispersive spectral lineshapes in the fingerprint region, which is a well-known effect in the CARS intensity as a result of the interference between the resonant and nonresonant part of the CARS susceptibility. Retrieved $\mathfrak{I}(\bar{\chi})$ spectra recover Raman lineshapes and exhibit the characteristic vibrational bands of neutral lipids, namely, the following: the $\mathrm{CH}_{2}$ and $\mathrm{CH}_{3}$ deformations at around $1450 / \mathrm{cm}$, the $\mathrm{C}=\mathrm{C}$ stretch band at $1660 / \mathrm{cm}$ (which is absent in saturated lipids), the $\mathrm{C}=\mathrm{O}$ stretch band at $1740 / \mathrm{cm}$ from the ester bonds between glycerol and the fatty acids, demonstrating the storage of lipids in the form of triglycerides, the peak at around $2850 / \mathrm{cm}$ from the $\mathrm{CH}_{2}$ symmetric stretch vibrational resonance, the broad shoulder at around $2930 / \mathrm{cm}$ which is a combination of $\mathrm{CH}_{3}$ stretch vibrations and $\mathrm{CH}_{2}$ asymmetric stretch enhanced by the broadening and shift of the $\mathrm{CH}$ deformations in the liquid phase, and the $=\mathrm{CH}$ stretch which gives rise to a band around $3010 / \mathrm{cm}$. The images show how cells increase the size of their cytosolic LDs over time, while maturing into adipocytes. The overall line shape of the $\mathfrak{J}(\bar{\chi})$ spectra remains mostly unaltered as the relative amplitudes between significant vibrational bands is approximately constant overtime. This suggests that, besides growing in size, no major changes occur in the chemical composition of LDs during maturation in the normal differentiation medium.

Different results are observed for cells grown in media supplemented with fatty acids. An example of hyperspectral CARS images and representative spectra as measured and after phase retrieval is shown in the Supporting Information (Figure S1) for human ADSCs cultured in LA-supplemented medium. The line shape of the $\mathfrak{I}(\bar{\chi})$ spectra does change over time, particularly for the bands at $1660 / \mathrm{cm}$ and $3010 / \mathrm{cm}$, which show an amplitude increase indicating the presence of more unsaturated $\mathrm{C}=\mathrm{C}$ bonds within LDs.

In order to obtain a more quantitative assessment of these time-course changes and gain an overview for all measured samples, for each group of cells fed with a given set of lipidsupplemented media at a given time, we determined the main lipid spectral component using the unsupervised $\mathrm{FSC}^{3}$ analysis (see Materials and Methods). We considered four separate chemical components in the factorization algorithm, which was the minimum number needed to well-represent the chemical composition of all samples. An example of this factorization with the corresponding spectra profiles and spatially resolved chemical maps of the components in vol/vol concentrations is shown in Figure 2 for a human ADSC grown in LA-supplemented media for $72 \mathrm{~h}$ after day 0 . The observed spatial and spectral profile enabled us to assign these components to the aqueous medium surrounding the cell (component 1 and 2), the lipid content (component 4, in excellent agreement with the spectrum measured for pure LA), and a cytosolic protein distribution (component 3). ${ }^{9}$

A comparison of the spectral profiles of the lipid component from the FSC $^{3}$ analysis on each group of cells is shown in Figure 3 for the fingerprint and $\mathrm{CH}$-stretch vibrational ranges. These spectra indicate that the chemical composition of the lipid content of cytosolic LDs changes with time and according to the fatty acid type in the media. The lipid component for the control cells matured in the standard differentiation medium has a spectrum very similar to that of GTO, consistent with previous observations from us suggesting that $\mathrm{OA}$ is compositionally the main fatty acid chain in the ADSC differentiation medium $^{10}$ (dotted lines are Raman spectra measured on bulk pure lipids for comparison ${ }^{9,22}$ ). Cells grown in media supplemented with polyunsaturated LA show a lipid component that with time develops increasingly strong bands at $1660 / \mathrm{cm}$ and $3010 / \mathrm{cm}$. A similar behavior is observed in cells grown in media supplemented with the polyunsaturated LNA, where the spectrum of the lipid component with time resembles the spectrum of pure GTL. Interestingly, reverting at day 3 from LNA-rich medium into OA-supplemented medium does recover a GTO-like lipid spectrum over time.

In order to further quantify these changes, a ratiometric analysis was applied to the spectral profiles in Figure 3. The ratios of $\mathfrak{\Im}(\bar{\chi})$ at $1660 / \mathrm{cm}$ relative $1450 / \mathrm{cm}$, at $2930 / \mathrm{cm}$ relative to $2850 / \mathrm{cm}$, and at $3010 / \mathrm{cm}$ relative to $2850 / \mathrm{cm}$ are plotted in Figure 4 for each group of cells at each time point. For reference, these ratios are also indicated for CARS measurements carried out on bulk pure lipids (GTO, LA, and $\alpha$-GTL) and analyzed with $\mathrm{FSC}^{3}$. It has been shown in the literature ${ }^{23}$ that a ratiometric analysis is useful to determine the degree of $\mathrm{C}=\mathrm{C}$ polyunsaturation and the order of lipids (i.e., their fluidity) in cytosolic LDs. In particular, the ratio of the phase-retrieved $\mathfrak{I}(\bar{\chi})$ between $1660 / \mathrm{cm}$ and $1450 / \mathrm{cm}$ was shown to provide a quantitative measure for the degree of lipid-chain unsaturation. ${ }^{23}$ 


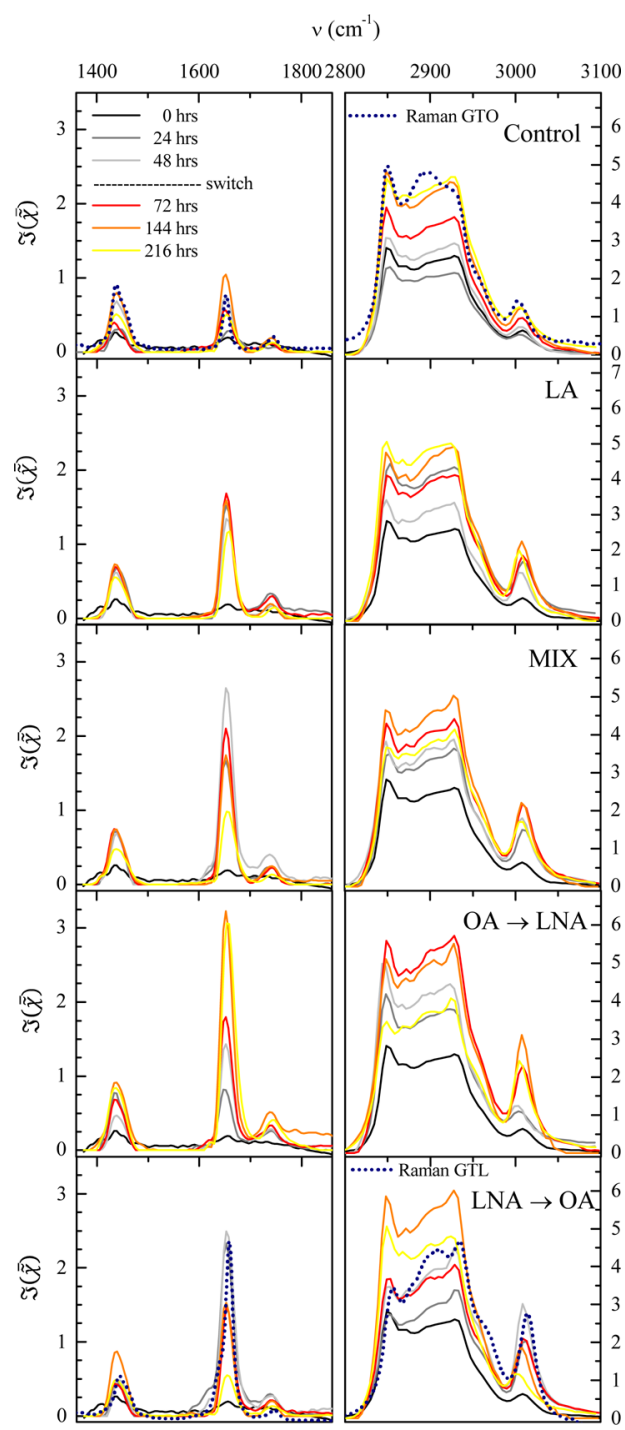

Figure 3. $\mathrm{FSC}^{3}$ lipid component on human ADSC grown in fatty-acid supplemented media over time, as indicated. The lipid component is blindly retrieved, following the factorization as shown in the example of Figure 2. Control: cells grown in the normal differentiation medium; LA: linoleic-acid-supplemented medium; MIX: 1:1 volume/ volume mixture of oleic acid and $\alpha$-linolenic acid; OA $\rightarrow$ LNA: on day 3 , the oleic-acid-supplemented medium was substituted with $\alpha$-linolenic-acid-supplemented medium; LNA $\rightarrow$ OA: on day 3 , the $\alpha$-linolenic-acid-supplemented medium was substituted with oleicacid-supplemented medium. Dotted lines are Raman spectra of bulk pure lipids for comparison.

The ratio of $\mathfrak{I}(\bar{\chi})$ between the band at around $2930 / \mathrm{cm}$ and the $\mathrm{CH}_{2}$ symmetric stretch resonance at $2850 / \mathrm{cm}$ can be used as a measure of the disorder, which usually correlates with the degree of unsaturation. $^{10,22}$ The ratio of the $3010 / \mathrm{cm}$ peak relative to $2850 / \mathrm{cm}$ is also a good measure of the degree of unsaturation. ${ }^{24}$ Figure 4 shows that cells grown in media supplemented with OA have a trend very similar to the control cells, again consistent with previous evidence that $\mathrm{OA}$ is compositionally the main fatty acid chain in the ADSC differentiation medium. ${ }^{10}$ The ratios are almost constant over the first $48 \mathrm{~h}$ time course in these cells, indicating no significant chemical change. Conversely, cells grown in media supplemented with LA or LNA show a rapid increase of the ratios, with $80-100 \%$ of the maximum value reached within the first $24 \mathrm{~h}$. For cells grown in

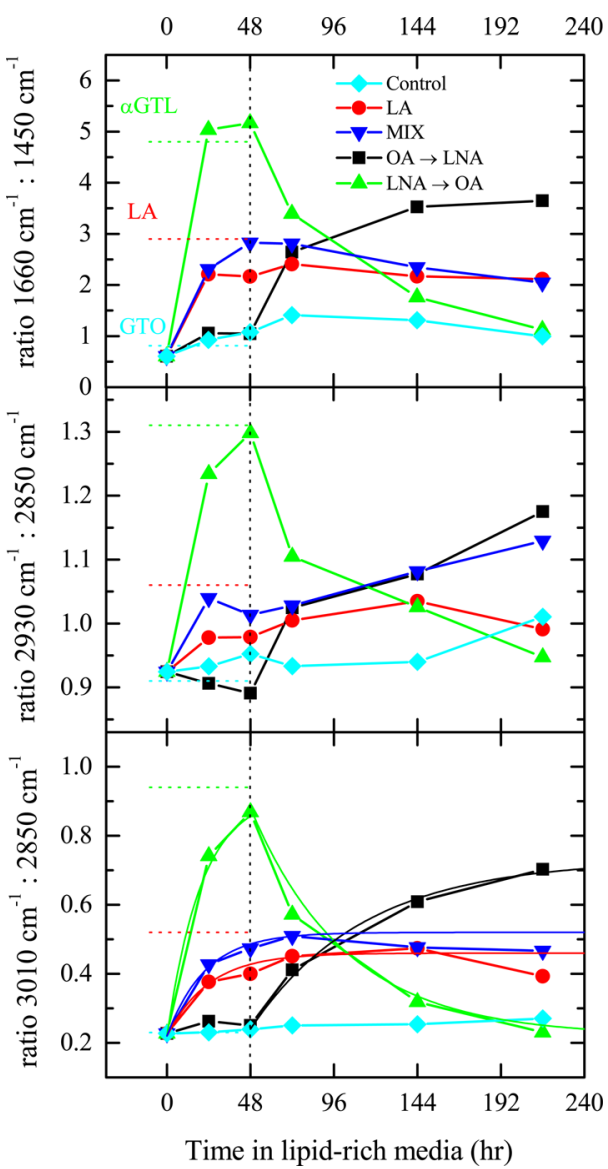

Figure 4. Ratiometric analysis of $\mathrm{FSC}^{3}$ lipid spectra shown in Figure 3. Upper panel: ratio between $1660 / \mathrm{cm}$ and $1450 / \mathrm{cm}$. Middle panel: ratio between $2930 / \mathrm{cm}$ and $2850 / \mathrm{cm}$. Lower panel: ratio between $3010 / \mathrm{cm}$ and $2850 / \mathrm{cm}$. Dotted lines indicated the ratios obtained from CARS measurements and $\mathrm{FSC}^{3}$ analysis carried out on bulk pure lipids (GTO, LA, and $\alpha$-GTL). Thin solid lines in the lower panel are exponential rise and decay functions, from which we deduce time constants of $22 \pm 2 \mathrm{~h}$ for the three samples grown over the first $48 \mathrm{~h}$ with LA-, LNA-, and equal mixture OA/LNA-supplemented media, and of $60 \pm 5 \mathrm{~h}$ when switching after $48 \mathrm{~h}$ to OA or LNA-rich medium.

LNA-supplemented media, reverting into OA-supplemented media after $48 \mathrm{~h}$ results in a decrease of these ratios, although at slower speed, and a recovery of the chemical composition into that of control cells after $216 \mathrm{~h}$, indicating reversible exchange. Furthermore, cells that have been matured first into OAsupplemented media over $48 \mathrm{~h}$ and then grown into LNAsupplemented media show uptake of the polyunsaturated fatty acids via an increase of the measured ratios; however, this occurs with a significantly slower speed than immature cells grown in LNA-supplemented media from day 0 , suggesting that the rapidity to respond to exogenous changes in lipid chemical content depends on the cell maturation stage. The time evolution of the $3010 / \mathrm{cm}$ to $2850 / \mathrm{cm}$ ratio is consistent with an exponential rise with a time constant of $22 \pm 2 \mathrm{~h}$ for the three samples grown over the first $48 \mathrm{~h}$ with LA, LNA, and equal mixture OA/LNA-supplemented media, as well as an exponential time constant of $60 \pm 5 \mathrm{~h}$ when switching after $48 \mathrm{~h}$ to OA or LNA-rich medium.

These dynamics are in good agreement with the time evolution of the lipid concentrations inside lipid droplets which we quantified as follows. Instead of applying the $\mathrm{FSC}^{3}$ analysis 


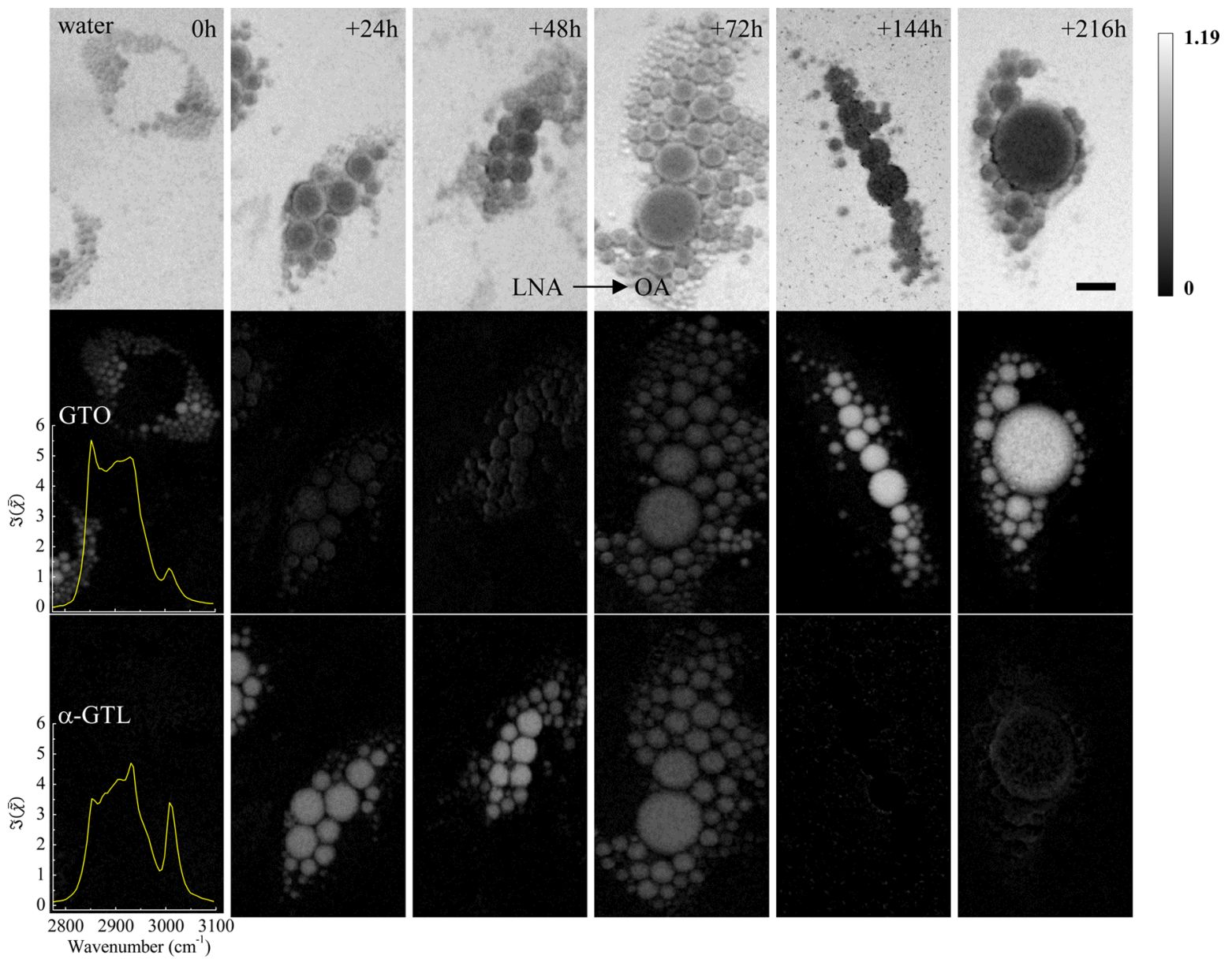

Figure 5. Comparative $\mathrm{FSC}^{3}$ analysis over the temporal data set of CARS hyperspectral images acquired in living ADSCs grown in a medium supplemented with LNA and at day 3 switched into a medium supplemented with OA. The spatial distributions of the volume concentration are shown at different time points for the three components of the analysis (water, glyceryl trioleate, and $\alpha$-glyceryl trilinolenate), on a grayscale as indicated. Scale bar is $10 \mu \mathrm{m}$. Retrieved spectra are shown for the lipid components. The concentration error in the analysis ${ }^{9}$ was found to be 0.05 on average with 0.04 standard deviation (see also Supporting Information Figure S2).

to individual set of cells separately at each time point, we applied the method to an entire temporal data set of hyperspectral images for a given group of cells grown in a supplemented medium, alongside the CARS hyperspectral images measured in the pure bulk triglycerides made of the fatty acids that were used in the supplemented medium. To ensure that the statistical weight of the images measured in pure triglycerides was appropriate for the analysis, these images were scaled to having effectively the same size (in terms of number of pixels) as those measured in cells. In this way, the concentration of the corresponding lipid type was identified inside lipid droplets over time. An example of the results from this analysis in the $\mathrm{CH}$-stretch region is shown in Figure 5 for cells grown initially in LNA-supplemented medium and at day 3 switched into OA-supplemented medium. In this case, the $\mathrm{FSC}^{3}$ analysis represents the overall chemical composition in terms of three components, namely, water, GTO, and $\alpha$-GTL (considering a fourth component either did not provide further relevant information or it caused instabilities and lack of reproducible convergence in the $\mathrm{FSC}^{3}$ algorithm). The lack of a protein component in this analysis is likely due to the significant statistical weight of the images of pure lipids, which resulted into the convergence of the $\mathrm{FSC}^{3}$ factorization algorithm toward these lipid species. Note that the protein component shown in Figure 3 is actually negligible inside LDs. The concentration maps of these three components over time are shown in Figure 5 in vol/vol units (see also Supporting Information Figure S2). A similar analysis was performed in the fingerprint region and showed consistent results (see Supporting Information Figure S3).

We further analyzed the concentration maps of the type shown in Figure 5 in order to extract the statistical distribution of the lipid concentrations over the ensemble of lipid droplets, at each time point and for each group of cells. This was achieved using an image segmentation in order to isolate regions containing only lipid droplets. The segmentation was performed semiautomatically using Cell Profiler. A typical threshold of $7.5 \%$ in the total lipid concentration was used to discriminate a LD from background and cytosolic lipids. To avoid that spatially close lipid droplets were assigned as a single object, we used an automatic algorithm which interprets the presence of local intensity maxima within the cluster as a signature of different droplets. We visually assessed the quality of this "declumping", and in unsatisfactory cases, manual declumping was performed. Once a LD was identified, the integrals of the two lipid components over the droplet area were calculated. The relative content $R_{\mathrm{i}}$ of one lipid component (i = GTO, LA, $\alpha$-GTL) for each droplet was then calculated by integrating the corresponding concentration $L_{\mathrm{i}}$ over the droplet area $A$ to obtain the lipid area $A_{\mathrm{i}}^{\mathrm{L}}=\int_{A} L_{\mathrm{i}} \mathrm{d} A$ and dividing by the total lipid area in that 

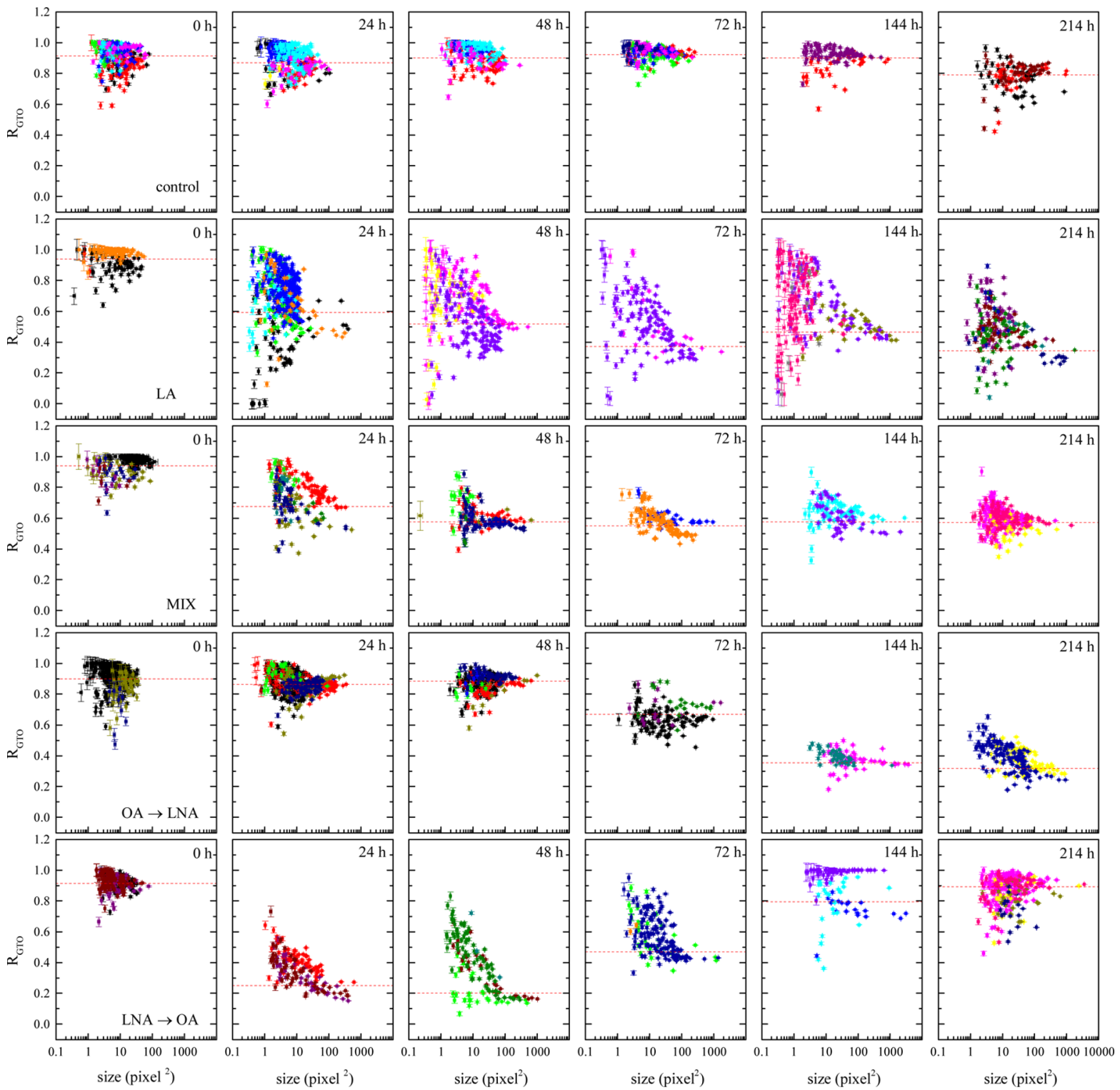

Figure 6. Relative content of GTO, $R_{\mathrm{GTO}}$, versus lipid area $A^{\mathrm{L}}$ (see text) for each droplet (pixel $=0.3 \mu \mathrm{m}$ ). Symbols of equal color correspond to droplets in the same cell. The time evolution for each cell group is shown from left to right as indicated. Red lines show the average GTO content $\bar{R}_{\text {GTO }}$ over all droplets.

droplet $A^{\mathrm{L}}=\sum_{\mathrm{i}} A_{\mathrm{i}}^{\mathrm{L}}$, so that $R_{\mathrm{i}}=A_{\mathrm{i}}^{\mathrm{L}} / A^{\mathrm{L}}$. The resulting $R_{i}$ are shown in Figure 6 on all samples for the GTO component versus $A^{\mathrm{L}}$. To elucidate differences in the time course between lipid droplets within one cell versus cell-to-cell heterogeneity, LDs of individual cells have been color coded $(2$ fields of view per time point per cell group were analyzed, resulting in 26 cells and hundreds of droplets per panel in Figure 6). Error bars in $R_{\mathrm{i}}$ and $A^{\mathrm{L}}$ are calculated from the corresponding values deduced in areas without lipid droplets, to indicate instrumental errors (note that we find 2 to 8 times smaller errors for data taken in the $\mathrm{CH}$ stretch region compared to data taken in the fingerprint region, which is due to the smaller $\mathfrak{I}(\bar{\chi})$ in the latter). We also verified that lipid droplet motion over the time scale of the image acquisition was not affecting this analysis. Including hyperspectral images measured in pure bulk triglycerides in the $\mathrm{FSC}^{3}$ analysis prevented the occurrence of retrieved lipid spectra distorted from motion artifacts. However, organelle motion manifested as a slight spatial shift between the retrieved chemical maps of different lipid components for a given lipid droplet. This effect is not significant when closely packed medium-large droplets are imaged, but it was more evident for small, faster moving droplets in the early stage of cell differentiation. We verified that this motion did not affect the quantitative analysis of $R_{\mathrm{i}}$ by eroding the segmented object areas by one pixel around the object contour and obtaining the same results as shown in Figure 6 within errors (see Supporting Information Figure S4). For cells grown in supplemented media after 24 and $48 \mathrm{~h}$, we observe a larger distribution of lipid contents than in the control case, which suggests a more heterogeneous lipid uptake. This is especially pronounced for smaller droplets and extremely evident for cells grown in LAsupplemented media (see Supporting Information Figure S5 for an overlay of the GTO and LA concentration maps at $144 \mathrm{~h}$ in this sample). At later times during differentiation, there is a general trend toward forming a more homogeneous distribution of lipid contents as compared to the earlier time points. Furthermore, when switching from OA-supplemented medium 
into LNA-supplemented medium at day 3, the lipid content appears slightly more homogeneously distributed than in the case of cells grown in LNA-rich medium from day 1 . Interestingly, we observe some cell-to-cell heterogeneity, but the qualitative behavior of the time course change in the lipid content for the overall population is followed at the single cell level. To the best of our knowledge, this time-dependent, size-dependent, and lipid-dependent heterogeneous uptake has not been quantified before and should bring new insights into the understanding of lipid metabolism in ADSCs.

As a measurement of the average lipid content, we use $\bar{R}_{i}=\sum A_{i}^{\mathrm{L}} / \sum A^{\mathrm{L}}$ summing over all droplets. $\bar{R}_{i}$ is shown by the red dotted line in Figure 6 for GTO and as an overall time course for all lipid components in Figure 7 (see Supporting

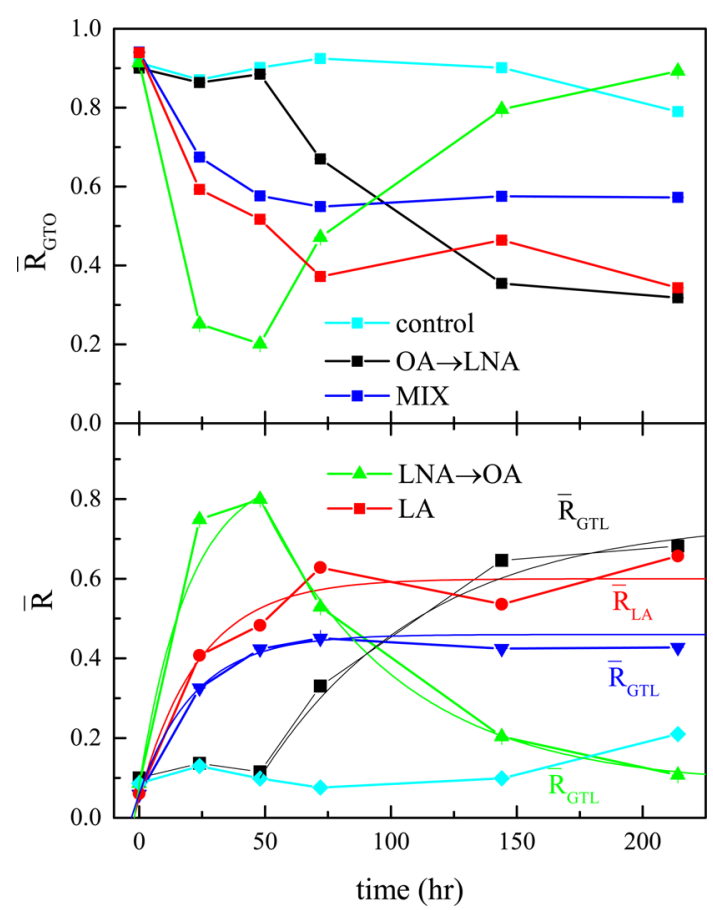

Figure 7. Average lipid content over time for each cell group. Thin solid lines in the bottom panel indicate monoexponential rise and decay functions, with the same time constants as those in Figure 4.

Information Figure S6 for the analysis after eroding the segmented object areas by one pixel all around the object contour showing no significant differences to the results in Figure 7). Thin solid lines in Figure 7 indicate monoexponential rise and decay functions, with the same time constants as those used for the $3010 / \mathrm{cm}$ to $2850 / \mathrm{cm}$ ratio in Figure 4, namely, a rise time of $22 \pm 2 \mathrm{~h}$ for $R_{\mathrm{LA}}$ and $R_{\mathrm{GTL}}$ in the three samples grown over the first $48 \mathrm{~h}$ with LA-, LNA-, and equal mixture OA/LNA-supplemented media, and a time constant of $60 \pm 5 \mathrm{~h}$ when switching after $48 \mathrm{~h}$ to OA or LNA-rich medium. We note that the process of initial lipid droplet formation and subsequent growth are thought to occur via distinct mechanisms (see recent review in ref 25). Our data reinforce this hypothesis, with evidence showing that initial lipid droplet formation and subsequent maintenance are differentially regulated and have distinct lipid trafficking kinetics.

\section{CONCLUSIONS}

In conclusion, we have shown that CARS hyperspectral imaging is suited to measure the time course of lipid uptake label-free and chemically specific at the individual droplet level in human adipose-derived stem cells during differentiation over 9 days after induction of adipogenesis in media supplemented with different fatty acids. Through the aid of an advanced quantitative data analysis method developed by us, we were able to distinguish lipid types and quantify their spatial distribution within and across lipid droplets, as well as their content in $\mathrm{vol} / \mathrm{vol}$ concentration units. We observed an heterogeneous uptake which was droplet-size dependent, time dependent, and lipid dependent, with the case of cells grown in linoleic-acidsupplemented media showing the largest distribution of lipid content at all times during differentiation. When analyzing the average lipid content, we found that adding new polyunsaturated lipids (linoleic and alpha-linolenic fatty acids) into the normal differentiation medium (mostly containing monounsaturated fatty acids) from day 0 resulted in uptake of the new lipid components with a $22 \pm 2 \mathrm{~h}$ exponential time constant, while switching lipids at day 3 resulted in an exponential time constant of $60 \pm 5 \mathrm{~h}$, suggesting that the rapidity to respond to exogenous changes in lipid chemical content depends on the cell maturation stage. Overall, these are unprecedented findings which should stimulate a better, more quantitative understanding of lipid metabolism in these cells. Besides being a model system for human adipogenesis, ADSCs are also attracting increasing attention in stem-cell-based therapies for the repair and regeneration of various tissues as alternatives to embryonic stem cells. ${ }^{26}$ Therefore, the quantitative imaging method shown here could pave the way toward a much better understanding of the differentiation capacities of ADSCs for applications in regenerative medicines. Furthermore, the field of lipid droplet-organelle interactions is only just emerging (reviewed in ref 27). Through the use of chemically specific CARS hyperspectral imaging of lipids, correlative with fluorescence microscopy of labeled organelles, it should become possible to explore this challenging area and to understand how specific lipids are delivered to organelles such as the endoplasmic reticulum, mitochondria, and peroxisomes in living cells for the first time.

\section{ASSOCIATED CONTENT}

\section{Supporting Information}

The Supporting Information is available free of charge on the ACS Publications website at DOI: 10.1021/acs.analchem.5b04468.

Hyperspectral CARS imaging on living ADSCs in LAsupplemented medium, additional data on combined FSC $^{3}$ analysis (PDF)

\section{Accession Codes}

Data access information about the data created during this research, including how to access it, is available from Cardiff University data archive at http://dx.doi.org/10.17035/d.2015. 0008101436.

\section{AUTHOR INFORMATION}

\section{Corresponding Author}

*E-mail: borrip@cf.ac.uk.

\section{Notes}

The authors declare no competing financial interest.

\section{ACKNOWLEDGMENTS}

The CARS microscope development was funded by the U.K. BBSRC Research Council (grant no. BB/H006575/1). C.D.N. acknowledges financial support by the President's Research 
Scholarship programme of Cardiff University and by the U.K. EPSRC Research Council (grant no. EP/I016260/1). P.B. acknowledges the UK EPSRC Research Council for her Leadership Fellowship Award (grant no. EP/I005072/1).

\section{REFERENCES}

(1) Schie, I. W.; Nolte, L.; Pedersen, T. L.; Smith, Z.; Wu, J.; Yahiatène, I.; Newman, J. W.; Huser, T. Analyst 2013, 138, 66626670.

(2) Zumbusch, A.; Langbein, W.; Borri, P. Prog. Lipid Res. 2013, 52, 615-632.

(3) Evans, C. L.; Potma, E. O.; Puoris'haag, M.; Côté, D.; Lin, C. P.; Xie, X. S. Proc. Natl. Acad. Sci. U. S. A. 2005, 102, 16807-16812.

(4) Saar, B. G.; Freudiger, C. W.; Reichman, J.; Stanley, C. M.; Holtom, G. R.; Xie, X. S. Science 2010, 330, 1368-1370.

(5) Camp, C. H., Jr.; Cicerone, M. T. Nat. Photonics 2015, 9, 295305.

(6) Wang, P.; Li, J.; Wang, P.; Hu, C.-R.; Zhang, D.; Sturek, M.; Cheng, J.-X. Angew. Chem., Int. Ed. 2013, 52, 13042-13046.

(7) Billecke, N.; Rago, G.; Bosma, M.; Eijkel, G.; Gemmink, A.; Leproux, P.; Huss, G.; Schrauwen, P.; Hesselink, M. K. C.; Bonn, M.; Parekh, S. H. Histochem. Cell Biol. 2014, 141, 263-273.

(8) Lee, Y. J.; Vega, S. L.; Patel, P. J.; Aamer, K. A.; Moghe, P. V.; Cicerone, M. T. Tissue Eng., Part C 2014, 20, 562-569.

(9) Masia, F.; Glen, A.; Stephens, P.; Borri, P.; Langbein, W. Anal. Chem. 2013, 85, 10820-10828.

(10) Di Napoli, C.; Pope, I.; Masia, F.; Watson, P.; Langbein, W.; Borri, P. Biomed. Opt. Express 2014, 5, 1378-1390.

(11) Masia, F.; Borri, P.; Langbein, W. Opt. Express 2014, 22, 40214028.

(12) Masia, F.; Karuna, A.; Borri, P.; Langbein, W. J. Raman Spectrosc. 2015, 46, 727-734.

(13) Karuna, A.; Masia, F.; Borri, P.; Langbein, W. J. Raman Spectrosc.

2016, in press, DOI: $10.1002 /$ jrs. 4876 .

(14) Jüngst, C.; Klein, M.; Zumbusch, A. J. Lipid Res. 2013, 54, 3419-3429.

(15) Mouras, R.; Bagnaninchi, P. O.; Downes, A. R.; Elfick, A. P. D. J. Biomed. Opt. 2012, 17, 116011.

(16) Lafontan, M.; Langin, D. Prog. Lipid Res. 2009, 48, 275-297.

(17) Hodson, L.; Skeaff, C. M.; Fielding, B. A. Prog. Lipid Res. 2008, 47, 348-380.

(18) Krahmer, N.; Farese, R. V.; Walther, T. C. EMBO Mol. Med. 2013, 5, 973-983.

(19) Listenberger, L. L.; Brown, D. A. Curr. Protoc. Cell Biol. 2007, $35,1-11$.

(20) Pope, I.; Langbein, W.; Watson, P.; Borri, P. Opt. Express 2013, 21, 7096-7106.

(21) Rocha-Mendoza, I.; Langbein, W.; Borri, P. Appl. Phys. Lett. 2008, 93, 201103.

(22) Di Napoli, C.; Masia, F.; Pope, I.; Otto, C.; Langbein, W.; Borri, P. J. Biophotonics 2014, 7, 68-76.

(23) Rinia, H. A.; Burger, K. N. J.; Bonn, M.; Müller, M. Biophys. J. 2008, 95, 4908-4914.

(24) Heinrich, C.; Hofer, A.; Ritsch, A.; Ciardi, C.; Bernet, S.; RitschMarte, M. Opt. Express 2008, 16, 2699-2708.

(25) Ohsaki, Y.; Suzuki, M.; Fujimoto, T. Chem. Biol. 2014, 21, 8696.

(26) Mizuno, H.; Tobita, M.; Uysal, C. Stem Cells 2012, 30, 804810.

(27) Barbosa, A. D.; Savage, D. B.; Siniossoglou, S. Curr. Opin. Cell Biol. 2015, 35, 91-97. 